\title{
Les médias dans la région : quels enjeux pour l'information locale?
}

\author{
Aimé-Jules Bizimana ${ }^{a}$
}

RÉSUMÉ. Avec l'actuelle crise des médias causée par la révolution numérique, les territoires locaux ont ressenti fortement les incidences des bouleversements des modèles d'affaires sur l'existence des salles de rédaction et sur la vitalité de l'information locale. Les effets combinés de la crise économique et de la crise de la publicité dans les modèles d'affaires ont été dévastateurs dans certaines régions. Cet article présente quatre axes de réflexion sur l'évolution des médias régionaux et locaux au Québec. Les enjeux économiques, politiques, de développement local et de déséquilibres entre les centres urbains et les collectivités régionales et rurales en lien avec les médias locaux se posent dans le contexte de l'essor du numérique et de l'intervention étatique par le biais des politiques publiques.

ABSTRACT. With the actual media crisis caused by the digital revolution, local territories have felt the impact of business models shifts on the existence of newsrooms and the vitality of local news. The combined effects of the economic crisis and the advertising crisis on business models have been devastating in some regions. This article presents four areas of reflection on the evolution of regional and local media in Quebec. The economic, political, local development and imbalance issues between urban centers and regional and rural communities in relation to local media arise with the advent of the digital media boom and state intervention through public policies.

\section{Introduction}

L'évolution des médias dans les régions est liée à des enjeux économiques, politiques et sociaux de différents ordres. Ces enjeux s'inscrivent dans la problématique générale de la démocratisation et du développement local des collectivités régionales. Le secteur médiatique est un espace de tension entre les territoires urbains et ruraux. La tension a trait aux questions d'accessibilité à l'information, de traitement et de circulation de l'information, de disponibilité des ressources techniques et économiques, de visibilité et d'attractivité de chaque territoire, etc. Quel est le rapport de la ruralité à ces enjeux?

Dans la situation médiatique actuelle, l'enjeu économique est de loin le plus urgent en raison de la crise des modèles d'affaires qui affecte les médias. La situation d'insolvabilité du Groupe Capitales Médias (GCM) et ses six quotidiens régionaux a causé un énorme choc. Cet enjeu lié aux ramifications de la révolution numérique vient prolonger celui lié aux conséquences de la concentration des médias et de la crise économique. La baisse des revenus et des effectifs touche les médias locaux dans un contexte de recul de l'information locale (Forum des politiques publiques, 2017, 2018) et de pauvreté de l'information locale (Lindgren, Corbett et Jaingris, 2017). Au Québec, la presse écrite régionale a été le secteur le plus touché par des fermetures (Brunelle et Brin, 2019). Les transformations en cours provoquent un remodelage économique majeur avec des restructurations dans le secteur régional du papier (Bizimana et Kane, 2019).

Dans la foulée de la crise des quotidiens régionaux, la Commission de la culture et de l'éducation, qui s'est dotée d'un mandat d'initiative sur l'avenir des médias d'information, a entamé ses consultations en vue d'établir un portrait médiatique global et de sonder les pistes de solution. Dans le contexte de la crise du marché publicitaire, de la domination des géants du Web et du déplacement des entreprises

\footnotetext{
a Professeur agrégé, Département des sciences sociales, Université du Québec en Outaouais et chercheur, Centre de recherche interuniversitaire sur la communication, l'information et la société (CRICIS)
} 
de presse vers l'univers multiplateforme numérique, l'enjeu économique apparait primordial, car il est lié à la survie même des médias. La question des aides publiques directes et indirectes (Brin et Descôteaux, 2018) ainsi que la taxation des géants américains sont au cœur du débat public.

Cet article présente quatre axes de réflexion qui éclairent la problématique des médias régionaux au Québec. De manière différenciée, chaque axe touche aux enjeux d'urbanité et de ruralité. En plus des enjeux économiques, cet article reviendra sur les enjeux médiatiques liés aux affaires publiques et au développement local. Au-delà de la logique de marchandisation, l'évolution des médias locaux a trait à la démocratisation municipale et à la question de la publicisation du débat démocratique. La relation difficile entre certains maires et les médias locaux constitue une manifestation dysfonctionnelle de la médiatisation. Les régions en appellent aux politiques publiques visant à renforcer leurs capacités de s'informer et d'informer en lien avec leur développement. L'importance de l'information locale dans une démocratie appelle à un renforcement des médias dans les collectivités en vue d'une gouvernance transparente.

Nous reviendrons sur certains constants relevés par la tournée régionale du Conseil de presse du Québec (CPQ, 2008) et par le rapport L'information an Québec: un intérêt public (ou rapport Payette; GTAJIQ, 2010). Les médias régionaux et locaux se définissent souvent par rapport aux médias nationaux, qui sont généralement plus nantis et dont les enjeux sont mieux couverts. Par leur statut et leur attrait, les métropoles accaparent généralement plus d'attention que les petites villes et les collectivités rurales. Nous verrons que les pressions économiques peuvent accentuer des déséquilibres centre/périphérie et urbanité/ruralité.

\section{Les enjeux économiques et numériques}

Dans les années 2000, des mouvements de concentration importants ont suscité de fortes inquiétudes dans les médias au Québec, particulièrement les journaux régionaux, dans un contexte où les grands groupes médiatiques cherchaient à se positionner sur un échiquier caractérisé par l'essor d'Internet et par l'ouverture à la concentration croisée (le fait de posséder des médias de plusieurs types : journaux, postes de radio, chaînes de télévision, portails Internet, etc.). Par la suite, la crise économique de 2008 a frappé de plein fouet les médias du monde entier en exposant particulièrement la vulnérabilité inhérente au secteur de la presse écrite. Le recul des ventes publicitaires occasionné par cette crise a fortement fragilisé tous les médias dont la publicité occupait une très grande part dans les recettes budgétaires. Certes, au moment de la crise, il n'y a pas eu au Québec d'hécatombe comme celle qu'on a vue aux États-Unis avec une cascade de fermetures des médias. Les médias nationaux ont été les plus touchés par la contraction publicitaire avec des impacts rédactionnels (Brin et St-Pierre, 2013), mais les répercussions de la crise ont été aussi senties dans les médias régionaux québécois avec, entre autres, des négociations difficiles avec les syndicats et certaines baisses d'effectifs. Un des éléments qui ont été très peu soulevés par la recherche et par les observateurs est l'impact de la crise économique sur les fonds de pension des médias.

Après les pressions économiques subies dans les années 2000, les médias n'ont pas eu le temps de souffler. La décennie 2010 a cristallisé les transformations économiques et sociales déjà en cours, puis a constitué un autre moment charnière avec une reconfiguration majeure de l'écosystème médiatique. La crise généralisée des médias repose sur les transformations liées à la numérisation de la société, lesquelles ont de sérieuses incidences sur leurs modèles d'affaires. L'émergence de nouvelles plateformes, de nouvelles habitudes de consommation et d'une culture de la gratuité fait partie des nouveaux déterminants de l'économie des industries culturelles. Dans les régions, le portrait territorial entre 2011 et 2018 révèle des fermetures et des difficultés surtout pour la presse écrite (Brunelle et Brin, 2019).

L'économie politique des médias régionaux est aujourd'hui marquée par une crise des modèles d'affaires qu'il faut entendre comme une crise de la publicité. L'écosystème médiatique régional connaît un remodelage structurel fondé sur des transformations induites par la révolution numérique. Ce remodelage se traduit par une crise dans le secteur du papier, dont les marges de profitabilité sont négatives. Trois retraits progressifs mais significatifs d'acteurs majeurs traduisent le mouvement de fond qui traverse le secteur du papier et 
qui affecte profondément la structure de propriété des médias régionaux (Bizimana et Kane, 2019) : le retrait de Québecor (cession de 74 hebdomadaires à Transcontinental Media en 2013), le retrait de Power Corporation (cession de 6 quotidiens régionaux par la filiale Gesca à Groupe Capitales Médias) et le retrait de Transcontinental (cession de 93 journaux locaux et régionaux au Québec et en Ontario par TC Media à plusieurs acquéreurs).

\subsection{L'onde choc et les solutions}

L'annonce du Groupe Capitales Médias (GCM) concernant l'amorce d'une procédure de faillite et ainsi la mise à l'abri des créanciers a provoqué la plus forte onde de choc jamais ressentie au Québec sur la crise des médias. Acquéreur du nouveau groupe en 2015, le propriétaire Martin Cauchon a aussitôt présenté sa démission. Face au cataclysme de la fermeture de 6 quotidiens régionaux sur 10, le gouvernement du Québec, par le biais d'Investissement Québec, a immédiatement octroyé un prêt de $5 \mathrm{M} \$$, le temps qu'un nouvel acquéreur se manifeste. Cette aide s'ajoutait alors aux 10 M \$ que GCM avait déjà reçus de Québec en 2017. Cette saga a démontré la fragilité particulière du modèle régional par rapport aux médias nationaux, même si la crise est généralisée.

Au cour de la tourmente, les travaux de la Commission de la culture et de l'éducation sur l'avenir des médias d'information ont débuté à point nommé. Lors des audiences de la commission, Claude Gagnon, le président-directeur général de GCM, a indiqué que, sur une période de 10 ans (2009-2019), le groupe régional a écopé d'une perte de $50 \%$ des revenus et d'une réduction du personnel de $53 \%$ (Gagnon, 2019a). Il a ajouté que le déficit actuariel des régimes de retraite atteignait $65 \mathrm{M} \$$. En cas de faillite, les employés actuels perdraient $25 \%$ de leurs prestations, tandis que les retraités écoperaient d'une coupure mensuelle de $22 \%$ à $25 \%$ (Vailles, 2019). Le plan de relance sous forme de coopératives exclut les régimes de retraite, un scénario qui fera perdre aux retraités jusqu’à $30 \%$ de leurs revenus.

Quand GCM a signalé l'intention de se placer sous la protection de la Loi sur la faillite et l'insolvabilité, le PDG Gagnon a souligné que la baisse des revenus résultait « de la concurrence féroce que livrent les géants des réseaux sociaux » (Gagnon, 2019b). Cet état de fait a été soulevé par plusieurs autres intervenants lors des audiences de la Commission de la culture et de l'éducation. Évoquant une « spirale vers le bas », François
Olivier, président et chef de la direction de TC Transcontinental, a commenté : «Le choc publicitaire est trop rapide et trop grand. L'arrivée des géants américains tels Google, Apple, Facebook et Amazon a porté un coup dur, et ça continue » (Olivier, 2019). Joueur important dans le secteur régional québécois avant d'amorcer un processus de désengagement de la presse écrite, TC Media, la filiale de TC Transcontinental, a connu depuis 2015 une baisse des revenus de $40 \%$ et une baisse de $50 \%$ pour le journal Les Affaires $^{1}$, avec comme résultante une réduction de $25 \%$ de son personnel éditorial (Marcoux, 2019).

Pour la presse écrite, les coûts du papier et d'impression sont considérables. Par ailleurs, les quotidiens et les hebdomadaires doivent verser des contributions à titre de compensation aux municipalités pour le recyclage du papier. Cette obligation reste un irritant en raison de l'augmentation significative des coûts de traitement ${ }^{2}$. Parlant $d^{\prime}$ '« un point de rupture» à ce sujet, Denis Tétrault, directeur général de RecycleMédias, organisme qui représente plusieurs médias, appelle au changement de la Loi sur la qualité de l'environnement (Tétrault, 2019).

Pour pallier les difficultés criantes des médias, diverses solutions ont été proposées par différents acteurs. Plusieurs des pistes de solution étaient déjà connues, mais les travaux de la commission ont offert l'occasion de les ramener dans l'actualité. Étant les principaux coupables identifiés quant à la ponction de la manne publicitaire et à leur position dominante, les géants d'Internet sont les premières cibles des mesures correctives. Les appels à leur taxation sont nombreux et insistants, mais les modalités d'application restent à venir ${ }^{3}$. L'inaction des gouvernements sur le front de l'équité fiscale semble néanmoins intenable, car le déséquilibre des revenus publicitaires dans l'économie politique numérique est tout simplement inacceptable. Le constat est bien documenté et il est accablant. Il faut agir contre la domination américaine sur un service public mondial qui a des répercussions très locales.

La question des aides publiques se trouve également au cœur des préoccupations des acteurs médiatiques. Les aides de l'État peuvent certainement offrir un répit dans le contexte délétère que traversent les médias, mais elles restent limitées et ne font pas l'unanimité, en raison des difficultés inhérentes à leurs modalités d'application et du principe même d'indépendance des médias. Les gouvernements provincial et fédéral 
ont annoncé des aides pour soutenir principalement la transformation numérique et l'information locale. Le gouvernement du Québec a d'abord consenti une aide de plus de $36 \mathrm{M} \$$ en insistant sur le soutien de la presse régionale. Les aides se sont également matérialisées sous forme de prêts ponctuels remboursables par le biais d'Investissement Québec. En octobre 2019, l'annonce d'une aide annuelle de $50 \mathrm{M} \$$ assortie d'un crédit d'impôt remboursable de $35 \%$ sur la masse salariale a constitué une grande bouffée d'oxygène $e^{4}$. Cette aide prolonge des mesures en cours, notamment un crédit d'impôt pour la transformation numérique des médias écrits. Les aides publiques aident l'ensemble des médias écrits, mais elles visent particulièrement les territoires régionaux qui sont très affectés par la crise.

Parmi les solutions avancées depuis longtemps par les médias, il y a l'instauration des crédits d'impôt ${ }^{5}$ sur la masse salariale et les coûts de production ainsi qu'un réaménagement du placement publicitaire gouvernemental dans les médias. La publicité gouvernementale constitue une avenue de financement indirect intéressante, mais cette option a été elle-même happée par les transformations du numérique. Au moment de sa création, une des demandes de la Coalition pour la pérennité de la presse d'information au Québec (CPPIQ) portait sur cet aspect : «Augmentation significative du budget gouvernemental à l'égard des placements publicitaires dans les journaux québécois, qui ne reçoivent pas leur juste part des investissements publicitaires de l'État» (CPPIQ, 2017, p. 14). Québec s'est engagé à réviser sa politique de placement publicitaire. Si le diagnostic est bien posé à ce sujet, c'est alors une question de cohérence des politiques publiques. Là aussi, la révision du placement publicitaire pourrait avoir un impact beaucoup plus significatif dans les territoires ruraux.

Sur le plan municipal, depuis 2017, la Loi visant principalement à reconnaître que les municipalités sont des gouvernements de proximité et à augmenter à ce titre leur autonomie et leurs pouvoirs (projet de loi no 122 ) dispense les villes et les MRC de l'obligation de publier leurs avis publics dans les journaux locaux ${ }^{6}$. Cette disposition a été fortement déplorée par les médias régionaux. La CPPIQ avait estimé une perte annuelle de $10 \mathrm{M}$, laquelle serait fatale pour certains dans les régions et les grands centres (CPPIQ, 2017). La crise des quotidiens régionaux a provoqué un débat plus large sur l'achat local de la publicité par les villes. La municipalité de Sherbrooke a dépensé plus de publicités chez Google et Facebook, qui sont plus efficaces dans le ciblage géographique et dans la mesure de l'impact que dans les médias traditionnels (Custeau, 2019).

Hebdos Québec a aussi proposé la mise en place d'un crédit d'impôt de $50 \%$ des dépenses applicables à l'achat de publicités locales comme incitatif pour les entrepreneurs locaux (Hebdos Québec, 2019). Par ailleurs, certains exhortent le gouvernement du Québec à ne pas reproduire le modèle urbain et à prendre en compte les particularités des régions: "Annoncer sur le Web, en région, n’a pas du tout le même impact qu'en milieu urbain, car la population y a plus difficilement accès, Internet étant souvent moins rapide, moins accessible ou moins performant » (MPVradio, 2019, p. 4). Dans une perspective plus large, cet aspect traduit une différence d'enjeu entre la ruralité et l'urbanité.

Lors des audiences de la Commission de la culture et de l'éducation, GCM a avancé l'idée de créer une régie publicitaire provinciale qui regrouperait tous les médias du Québec et qui aurait une plus grande force dans le but de concurrencer les grandes compagnies (Gagnon, 2019b). Ce type d'initiative médiatique serait en effet une synergie rare entre les territoires urbains et ruraux en vue d'affronter une conjoncture numérique difficile. Sylvain Chamberland, président et fondateur d'Arsenal Media, a également soutenu la création d'une régie publicitaire en vue d'unir les forces, mais il a aussi proposé « un crédit d'impôt aux entreprises qui soutiennent les médias indépendants en prenant de la publicité numérique dans un média local » (Chamberland, 2019). La presse écrite est le secteur médiatique le plus touché par la crise, mais la radio et la télévision, même si elles ne sont pas à un point critique, subissent également les contrecoups de la migration publicitaire généralisée vers le Web. En commission parlementaire, Michel Lorrain, président de Cogeco Média, a fait savoir que :

L'industrie [de la radio] a conservé une certaine stabilité grâce, notamment, à des réductions de dépenses particulièrement en matière de programmation, de production et en administration de frais généraux. Mais, malgré tout, l'industrie est en perte de vitesse. Cela n'augure rien de bon pour l'avenir de la programmation parlée et des nouvelles radiophoniques locales dans les marchés régionaux (Lorrain, 2019).

En télévision, les revenus sont également généralement à la baisse. Depuis 2014, les marges bénéficiaires 
de la télévision privée commerciale sont négatives (Institut de la statistique du Québec, 2019). Pour la télévision publique, les stations régionales de RadioCanada ont principalement subi les pressions financières liées aux compressions du gouvernement Harper en 2014 et à l'élimination par le CRTC du Fonds pour l'amélioration de la programmation locale (FAPL), ce qui a entraîné une diminution des services régionaux. Le FAPL avait été créé en 2009 pour soutenir la programmation locale dans les petits marchés à la suite de la crise financière, mais le CRTC, jugeant que la situation économique s'était améliorée pour les stations de télévision, a aboli ce fonds. Le fonds avait été très mal reçu par les entreprises de distribution de radiodiffusion (EDR). Si l'on considère le financement jusque-là obtenu par le radiodiffuseur public, ce sont les stations régionales de Radio-Canada qui ont grandement souffert de l'abolition du fonds.

Dans le cadre des aides de l'État à la presse, les médias communautaires, qui vivent dans une situation de précarité permanente, ont vu leur financement augmenter en vue de soutenir la production d'une information de proximité. Par ailleurs, les associations des médias communautaires (presse écrite, radio et télévision) ont reçu une enveloppe de 400000 \$ pour mettre sur pied un laboratoire collaboratif d'innovation qui financera des projets de formation et de recherche-développement.

Les régions rurales possèdent un plus grand nombre de médias communautaires. Le placement publicitaire reste là aussi un enjeu important, car le secteur communautaire rappelle régulièrement le manquement récurrent du gouvernement du Québec à respecter le seuil de placement de $4 \%$ qu'il s'est fixé pour les médias communautaires. Là aussi, la cohérence du gouvernement doit être de mise pour soutenir un secteur qui contribue énormément à l'information régionale et locale ainsi qu’à l'identité de la ruralité.

\section{Les enjeux politiques}

Une société démocratique repose sur les piliers que sont la liberté de presse et le droit du public à l'information. Les médias participent au processus de démocratisation municipale en jouant le rôle de lien social et de contrepoids aux lieux de pouvoir de quelque nature. L'information locale est donc un intérêt public au cœur de la gouvernance et du fonctionnement démocratiques des territoires urbains et ruraux. À la suite de la crise des quotidiens régionaux, les intervenants aux travaux de la Commission de la culture et de l'éducation ont souligné l'importance des médias dans une société démocratique. Les expressions suivantes ont été utilisées : " élément fondamental de la démocratie», " chien de garde de la démocratie », "vecteur de démocratie », "préservation de la démocratie » et «fondement pour la démocratie dans les régions » (Assemblée nationale du Québec, 2019). La disparition des médias régionaux est alors associée à « un déni de la démocratie » ou à « une démocratie qui recule » dans des territoires locaux.

Il existe un lien indéniable entre les médias locaux et les affaires publiques dans les régions métropolitaines et dans les municipalités rurales. Ce lien se traduit à travers la couverture des débats publics locaux et l'influence que celle-ci est susceptible d'avoir sur la formation de l'opinion publique dans les collectivités urbaines et rurales et sur les décisions politiques des élus locaux. « Il n'y a pas de vie démocratique locale possible sans les médias régionaux ", a rappelé Maxime PednaudJobin, maire de Gatineau, lors d'un colloque sur l'information (Bélanger, 2017). La crise des quotidiens a suscité un élan de solidarité qu'on n'avait pas vu souvent et beaucoup d'élus locaux se sont portés à la défense du rôle des médias régionaux. Selon Paul Sarrazin, maire de Sainte-Cécile-deMilton et préfet de la $\mathrm{MRC}^{7}$ de la HauteYamaska :

Ce sont à travers ces médias que les débats naissent, que se crée une agora populaire où les citoyens peuvent émettre leurs idées. Où la politique locale est démocratisée, entendue par le plus grand nombre et où elle peut ainsi prospérer et demeurer transparente (Sarrazin, 2019, p. 10).

La couverture médiatique des débats locaux pose cependant certains défis. La relation entre le politique et les médias locaux est appelée, par nature, à être parfois conflictuelle, indépendamment des territoires urbains ou ruraux. De manière générale, les journalistes parviennent à réaliser leurs reportages sur les enjeux régionaux et locaux grâce au concours des sources en fonction publique dans les municipalités. Cependant, de nombreuses zones de friction existent. La relation entre certains maires et les journalistes fait régulièrement la manchette dans l'actualité. L'interaction entre les 
journalistes et les responsables locaux oscille entre collaboration et conflit. Comme ailleurs dans d'autres institutions, certains élus locaux reprochent aux médias, d'une part, d'être des empêcheurs de tourner en rond quant à l'avancement de certains projets municipaux et, d'autre part, de verser dans le sensationnalisme.

Des cas d'entrave, d'hostilité ou de représailles contribuent à créer la tension dans la relation entre les élus, dont certains se comportent comme des roitelets (Martel, 2018), et les «méchants journalistes » (Bourgault-Côté, 2017). La Fédération professionnelle des journalistes du Québec (FPJQ) dénonce cette situation depuis des années. Dans son dossier noir sur l'information municipale au Québec, la FPJQ a constaté une méconnaissance du travail journalistique et une hostilité envers les journalistes par certains élus (FPJQ, 2010). Les problèmes semblaient plus apparents dans les régions où les partis politiques avaient raflé une majorité lors des élections :

À force de questionner le travail des élus, au nom de l'intérêt public, les journalistes finissent par être confondus avec l'opposition dans ces villes où le maire règne sans partage. Leurs conditions de travail sont pénibles. Ils rencontrent des obstacles injustifiables que nous regroupons en trois grandes catégories : limites dans l'accès à l'information; mesures d'intimidation verbale, entraves et menaces; mesures de rétorsion économique (FPJQ, 2010, p. 3).

Ce constat sur les relations difficiles est également celui du Groupe de travail sur le journalisme et l'avenir de l'information au Québec (GTJAIQ, 2010a; Comeau, 2010).

La FPJQ rappelle régulièrement l'importance de la transparence de l'administration régionale et défend, à ce titre, la nécessité pour les élus et les fonctionnaires locaux de faciliter le travail des médias. Dans cette optique, la question de l'accès à l'information dans les administrations municipales reste encore un dossier litigieux entre les médias et les administrations régionales et locales. La FPJQ a recommandé que les municipalités soient assujetties au régime de la divulgation automatique (FPJQ, 2013). Ce changement nécessiterait une modification de la Loi sur les cités et villes, comme l'a recommandé également le rapport
Payette (GTJAIQ, 2010a). La portée des dispositions des lois sur l'accès à l'information est un enjeu récurrent sur les plans fédéral et provincial. Une révision de la loi québécoise en rapport avec les réalités médiatiques régionales se fait encore attendre.

La médiatisation des conseils municipaux constitue une autre pierre d'achoppement entre les responsables municipaux et les représentants des médias. La présence des journalistes et des caméras ainsi que l'enregistrement audiovisuel des séances de conseil indisposent certains maires, qui n'hésitent pas à invoquer le règlement sur l'ordre et le décorum pour expulser les médias. "L'existence de pratiques inégales, aléatoires ou arbitraires dans les municipalités fait en sorte que la règle de la publicité des débats n'est pas appliquée uniformément », déplore la FPJQ (2010, p. 20). Certains élus restent encore réticents à autoriser l'enregistrement des conseils municipaux, sous prétexte de maintenir le décorum, tandis que les médias réclament un accès aux séances publiques en invoquant la liberté de presse et le droit du public à l'information. Les réticences de certains maires sont liées entre autres à ce qu'on peut appeler l'« effet Infoman ${ }^{8}$ », qui traduit la crainte d'être ridiculisé sur un grand réseau de télévision ou sur les réseaux sociaux à la suite de situations susceptibles de provoquer des railleries.

En 2015, la Fédération québécoise des municipalités (FQM) a adopté une résolution invitant ses membres « à ne pas interdire l'enregistrement de leurs séances de conseil par les médias et à donner libre accès aux journalistes à leurs réunions » (FQM, 2015). En avril 2017, le commissaire aux plaintes du ministère des Affaires municipales et de l'Occupation du territoire a rappelé à Val-David que les citoyens avaient le droit d'enregistrer les séances du conseil (Villeneuve, 2017). En août 2017, des citoyens de LaSalle ont déposé en Cour supérieure un pourvoi en contrôle judiciaire dans le but de rendre invalide le règlement municipal interdisant la captation vidéo ou tout enregistrement des séances publiques du conseil d'arrondissement (Normandin, 2017). L'article de Normandin (2017) rappelle qu'en 1984, la Cour supérieure avait déjà donné raison à un citoyen de la municipalité de Saint-Sulpice à la suite d'un recours afin de faire invalider un règlement qui interdisait de filmer et d'enregistrer une séance publique. 
La publicisation des conseils municipaux est donc une question récurrente dans les relations entre les élus régionaux et les médias locaux. L'avenue juridique constitue une option pour la FPJQ (HoudeRoy, 2017), même si elle n'est pas souhaitable. Plusieurs pensent que seule une intervention de Québec à travers un mécanisme contraignant pour les municipalités règlera cette question une fois pour toutes.

Certaines villes diffusent les séances du conseil municipal par le câble, par webdiffusion en direct ou en différé. Les séances tenues à huis clos peuvent représenter un défi quant à la confidentialité des propos. Cependant, pour la FPJQ, « la webdiffusion ne remplace pas la presse et [il] ne faudrait pas qu'elle soit un prétexte pour interdire aux journalistes d'assister aux conseils municipaux » (Bélanger, 2017). La webdiffusion est plus utilisée dans les plus grandes villes (p. ex., région métropolitaine de Montréal) que dans les municipalités de 30000 habitants et moins. Elle est à peu près inexistante dans celles de moins de 7000 habitants (Bélanger, 2017). Au Québec, la ruralité accuse un retard par rapport aux territoires métropolitains.

La couverture médiatique des questions autochtones est un enjeu politique d'un autre ordre, mais qui touche à la fois les médias nationaux et régionaux. La tournée régionale du Conseil de presse du Québec en 2008 a évoqué brièvement la méconnaissance des Premières Nations: "Les citoyens québécois ne sont pas tous initiés à l'histoire des Premières Nations et ignorent souvent les principaux enjeux auxquels elles sont confrontées. Plusieurs préjugés persistent et la presse a un important rôle d'éducation à jouer » (CPQ, 2008). Les représentants autochtones dans les régions ont dénoncé une image médiatique des Autochtones souvent incomplète ou stéréotypée (Carignan, 2012). Ce constat sur une vision empreinte de préjugés et sur le manque de représentation a été réaffirmé avec force par l'Enquête nationale sur les femmes et les filles autochtones disparues et assassinées. Le rapport a conclu à une représentation erronée et à la sous-représentation des Autochtones dans les médias :

Les médias ne dépeignent pas correctement les femmes et les filles inuites, métisses et des Premières Nations, ni les personnes
2ELGBTQQIA9 en particulier. Par conséquent, ils entretiennent des stéréotypes négatifs à leur sujet. Ces stéréotypes perpétuent le racisme, le sexisme, l'homophobie, la transphobie et la misogynie à l'égard des femmes, des filles et des personnes 2ELGBTQQIA autochtones dans la population canadienne en général (ENFFDA, 2019a, p. 432).

Selon le rapport, la représentation médiatique inégale entre Autochtones et non-Autochtones est fondée sur des considérations de race et de classe, tout en escamotant la question des inégalités sociales et la violence symbolique consécutive au passé colonial. Une des recommandations enjoint aux « gouvernements d'offrir un financement et un soutien adéquats aux initiatives dirigées par les Autochtones et destinées à améliorer leur représentation dans les médias et la culture populaire » (ENFFDA, 2019b, p. 203).

\section{Les enjeux de développpement local}

Les médias sont plus souvent présentés comme des vecteurs de démocratie, mais moins souvent comme des vecteurs de développement. Dans un rare ouvrage de la Banque mondiale consacré au rôle des médias dans le développement économique, on note que « l'action des médias entraîne des retombées économiques, politiques et sociales »à travers l'influence sur le processus politique et la politique d'entreprise (Islam, 2005, p. 36). Dans les régions, les médias peuvent agir comme des acteurs du développement local avec des effets directs et indirects. Le lien entre un média et le développement local s'inscrit dans l'ancrage du média dans un territoire spécifique (urbain ou rural) et dans le rôle de reflet de la communauté qu'il joue. Les médias créent le lien social, et sont vecteurs de l'identité des villes et de la ruralité.

Combien pèse le secteur médiatique dans l'économie d'une région? Peu de données existent au Québec et au Canada sur le poids des médias dans les économies locales et régionales pour répondre à cette question. "La contribution directe des médias à la production économique est modeste; pratiquement dans toutes les économies, riches ou pauvres, c'est un secteur d'activité relativement restreint », note Owen (2005, p. 215) dans l'étude de la Banque mondiale. Certaines données sur la 
presse écrite avancent des retombées fiscales et des effets sur l'emploi sur les plans fédéral, provincial et municipal (MCE Conseils, 2016). Dans l'étude de Deloitte sur la contribution du radiodiffuseur public à l'économie canadienne, il est indiqué que "par ses dépenses à l'échelle du pays [3,56 G\$], CBC/Radio-Canada a un impact positif dans chaque province et territoire » (Deloitte, 2014, p. 2). Une étude sur la ville de Saguenay présente ce type de retombées positives pour l'industrie du cinéma, à laquelle contribuent aussi les radiodiffuseurs (Trigone, 2014). Il faut dire à ce sujet que les statistiques régionales à l'échelle des municipalités manquent cruellement.

Le lien entre les médias et le développement local est un pan oublié par la recherche universitaire et mériterait certainement une plus grande attention. Les villes et les acteurs ruraux en donnent certains indices. Certains plans stratégiques municipaux réfèrent clairement aux médias locaux comme des partenaires du développement local. Lors des consultations sur l'information d'intérêt public dans les médias en 2011, le mémoire de la Conférence régionale des élus de l'Abitibi-Témiscamingue a rappelé trois orientations du plan stratégique:

1. Favoriser une meilleure couverture médiatique ainsi qu'une meilleure connaissance des actualités de l'Abitibi-Témiscamingue, du Nord-du-Québec et du Nord-Est ontarien; 2. Favoriser les débats sur les enjeux locaux et régionaux; 3. Définir l'apport des médias de proximité dans le développement local et régional (CREAT, 2011, p. 4).

Le mémoire a également souligné le rôle des médias communautaires : « À titre de vitrine sur le milieu, ils participent au ciment qui définit l'identité d'un territoire et d'une région" (CREAT, 2011, p. 14). De manière générale, les métropoles ont une densité qui facilite l'implantation des médias, alors que les milieux ruraux sont moins desservis par les grands médias. Cette situation a un impact sur la visibilité médiatique du développement local dans l'espace de la ruralité.

La crise des médias régionaux au Québec a interpellé plusieurs acteurs politiques, économiques et sociaux, qui ont exprimé un soutien notable, mais aussi des inquiétudes par rapport à la vitalité de leur région. "L'enracinement local de ces médias génère une mobilisation locale d'égale mesure et l'enjeu est correctement perçu comme étant (aussi) un de développement régional » (Alain et Lalande, 2019). Des acteurs du milieu communautaire en Estrie abondent dans ce sens :

Nos médias locaux et régionaux sont des liants de nos communautés. Ils mettent en valeur les actions citoyennes, ils nous renseignent sur les bons coups et les événements qui ont cours dans les différents milieux, ils contribuent au sentiment d'appartenance envers notre région, notre municipalité, notre quartier. Ils sont le reflet de notre contribution collective et individuelle au développement social, communautaire et économique (Bibeau et Cyr, 2019).

Lors du colloque Information régionale et développement économique : Qu'en est-il dans votre région? en 2001, plusieurs intervenants régionaux ont souligné le rôle des médias dans la promotion de l'identité et de la fierté dans les collectivités ainsi que dans l'appropriation de la région par les médias (Demers et Giroux, 2001). Le maire de SaintGeorges de Beauce a pointé le double effet d'influence des médias :

À court terme, s'ils luttent contre un projet, celuici a peu de chances de se réaliser; à plus long terme et de manière indirecte, par le choix qu'ils accordent à telle ou telle nouvelle, ils influent sur les valeurs des communautés et donc sur les projets qui découlent de ces valeurs (Demers et Giroux, 2001).

Les acteurs du développement ont recours aux médias pour faire circuler l'information et comptent également sur eux pour la sensibilisation sur les enjeux de développement (Gilbert, Carrier et Proulx, 2002). La radio communautaire CKBN, dans la région Nicolet-Yamaska-Bécancour, a été citée par l'organisme Territoires innovants en économie sociale et solidaire (TIESS) comme exemple de partenariat entre les entreprises d'économie sociale et les municipalités, en soulignant entre autres que la promotion de la radio d'organismes et de commerces de la région a favorisé entre autres l'achat local (TIESS, 2014).

Par ailleurs, il faut noter aussi que le Programme d'aide au fonctionnement pour les médias communautaires du ministère de la Culture et des Communications s'inscrit plus largement dans les 
orientations de différentes politiques gouvernementales qui visent à favoriser la participation citoyenne à la vie collective et à sensibiliser la population au développement durable (MCC, 2013). «En soutenant les médias écrits, les radios et les télévisions communautaires de toutes les régions du Québec, le Ministère souhaite agir contre la concentration de la presse et favoriser le développement local et régional. Ces organismes sont vus comme des agents de cohésion sociale dans leur milieu » (MCC, 2013, p. 5).

Dans les régions, les médias peuvent aussi contribuer au rayonnement de différents secteurs locaux (culture, musique, tourisme, sport, etc.). Par leur médiatisation, les médias régionaux créent de la visibilité pour des projets de développement social et économique. On peut penser entre autres au rôle joué par les médias locaux et communautaires dans la visibilité des artistes et des talents locaux. Les stations régionales de radio et de télévision ainsi que les plateformes web ${ }^{10}$ contribuent grandement à la visibilité des contenus culturels locaux à l'échelle de la province. La journaliste Marie-Ève Martel souligne que « la valeur la plus importante des médias régionaux est leur capacité d'être le miroir des communautés qu'ils desservent» (2018, p. 31). Parmi ses principes directeurs, la Politique nationale de la ruralité mentionne d'sencourager la participation citoyenne » (MAMROT, 2013, p. 29). Cependant, il n'est nulle part fait mention du rôle que peuvent jouer les médias locaux dans la publicisation des politiques publiques et des réalités territoriales. Une gouvernance territoriale démocratique ne peut faire l'économie de la médiation des professionnels de l'information. Ainsi, le développement local a besoin de la médiatisation des projets structurants au cœur de la ruralité. Les territoires existent aussi à travers les récits médiatiques ${ }^{11}$.

Certes, les municipalités aiment quand les médias soulignent leurs bons coups, et plusieurs élus et autres acteurs conçoivent leur relation avec la presse à travers ce prisme. L'incompréhension ou l'inconfort surviennent néanmoins quand les projecteurs attirent l'attention sur des échecs et des scandales. Le rôle des médias dans une société qui aspire à l'idéal démocratique ne peut pas être rempli si la couverture ne porte pas sur les deux côtés de la médaille. Par leur médiation, les médias rapportent l'information d'intérêt public sur les affaires publiques et jouent un rôle d'accélérateur de la participation citoyenne. Même si les instances de médiation se multiplient à l'ère numérique, la fonction médiatique reste primordiale dans l'émergence d'un espace public régional et rural qui est porteur d'enjeux politiques, économiques et sociaux au cœur de l'action et du développement des collectivités territoriales.

\section{Les déséquilibres centre/périphérie}

L'univers des médias régionaux repose sur un certain nombre de déséquilibres qui témoignent de certaines différences et d'un rapport contrasté entre les médias des grands centres et ceux des territoires locaux et ruraux. Ces déséquilibres ont trait à la production et au traitement de l'information ainsi qu'à l'accès à certaines ressources techniques et financières. L'absence d'organes d'information dans un territoire donné constitue un cas limite de pauvreté médiatique.

Au Québec, le phénomène de la «montréalisation» de l'information est un trait récurrent du débat sur l'état des médias régionaux. Parlant d'un terme imparfait, Corriveau et Sirois (2012, p. 4) l'ont défini comme une "réduction du débat politique aux préoccupations des grands centres ». Ce phénomène se décline sous forme de déséquilibres différenciés. Le premier est un déséquilibre national/régional. La tournée régionale du Conseil de presse du Québec a soulevé cet aspect en pointant la surreprésentation des informations montréalaises dans les médias: «Les sièges sociaux des médias se concentrant principalement dans la métropole, ils occulteraient en grande partie l'information régionale et celle en provenance des grands centres urbains comme Québec ou Gatineau » (CPQ, 2008, p. 19). La montréalisation a été souvent critiquée dans le contexte de la concentration, où les entreprises de presse procèdent à un regroupement d'activités pour réaliser des économies d'échelle. Cependant, une étude a démontré que $10 \%$ de l'information dans Le Quotidien, Le Soleil et La Tribune en 2007 provenait du journal montréalais La Presse, alors au sein du groupe Gesca ${ }^{12}$, mais que cette proportion n'avait pas contribué au recul de l'information locale mais plutôt au recul des textes d'agences (Giroux et Marcotte, 2009).

Les médias nationaux ne parlent pas assez des réalités régionales et locales. «Le manque de représentations des réalités régionales dans les grands 
médias est aussi lié au manque de journalistes des médias nationaux en région» (Carignan, 2012, p. 53). Selon le rapport du Conseil de presse du Québec, la sous-couverture des régions entraîne un manque d'attrait dommageable pour la rétention des jeunes et pour le recrutement des entreprises, en plus d'engendrer une absence des régions à l'agenda politique, très influencé par les médias (CPQ, 2008). L'absence des régions dans l'actualité nationale se traduit également par l'absence médiatique des maires, qui sont les porte-voix des régions. La firme Influence Communication (2015) a calculé que $91 \%$ de l'intérêt médiatique pour les régions en 2015 portait sur Montréal, Québec et Saguenay, avec une attention démesurée pour les maires Denis Coderre, Régis Labeaume et Jean Tremblay, qui avaient une forte personnalité.

Le déficit d'information réel ou potentiel en défaveur des milieux locaux crée des préoccupations réelles. Quand le réseau radiophonique Corus a indiqué que l'émission matinale produite à Montréal allait être diffusée dans les stations régionales du groupe en 2010, plusieurs critiques ont été entendues. «Il s'agit d'un soufflet pour les auditeurs de CJRC 104,7 FM, en Outaouais, et des trois autres régions qui subiront le même lavage "montréaliste", l'Estrie, la Mauricie et le Saguenay. [...] Cette émission ne fera que parler À l'Outaouais. Elle ne parlera pas DE l'Outaouais » (Jury, 2010, p. 10).

Abondant dans le même sens, l'Association des radiodiffuseurs communautaires du Québec note que la concentration des médias favorise l'uniformisation des messages et des modes de pensée, et véhicule une vision urbaine avec des prises de décision sur les contenus qui proviennent des grands centres (ARCQ, 2016). La centralisation de la production de tous les bulletins régionaux du Groupe TVA à Montréal a suscité les mêmes craintes (Baillargeon, 2016). Le CRTC a déjà également reconnu « la montréalisation des ondes » de la télévision traditionnelle en appelant un meilleur reflet des non-Montréalais (CRTC, 2012).

Le déséquilibre national/régional se prolonge à travers un clivage régional/local ou urbain/rural. La tournée du Conseil de presse du Québec a également souligné les manques dans la couverture des régions rurales par rapport aux grands centres :

Le territoire des différentes régions administratives est parfois très étendu et requiert que les journalistes parcourent d'importantes distances pour couvrir toutes les informations régionales, ce qui est souvent impossible et qui occasionne une concentration de la couverture vers les centres urbains régionaux (centralisation de l'information en région) et une faiblesse de couverture en périphérie et dans les milieux ruraux (CPQ, 2008, p. 9).

L'aspect territorial est également souligné par le rapport Payette : «L'immensité du territoire québécois est une réalité qu'il ne faut pas négliger et qui freine la couverture journalistique régionale » (GTJAIQ, 2010a, p. 60). De manière générale, on peut dire que plus une ville est densément peuplée, mieux elle est desservie par les médias, toutes catégories confondues.

De la même manière qu'on évoque la «montréalisation » pour signifier le clivage national/régional, ailleurs, on parle aussi de "sherbrookisation » pour nommer «le manque de couverture de la grande région et la concentration sur Sherbrooke » (CCE, 2006, p. 5). Il convient mieux alors de parler de « métropolisation de l'information ». Ce terme peut être défini comme une couverture médiatique où les sujets, les enjeux ou les moyens de production provenant des grands centres urbains sont surreprésentés par rapport à ceux des territoires locaux. Dans cette définition, il est plus approprié de parler de territoires locaux que régionaux. La tournée du Conseil de presse du Québec a en effet fait écho d'un déséquilibre centre/périphérie, qui s'exprime à l'intérieur même des grands centres :

Les habitants de Montréal et des grands centres urbains n'ont pas l'impression de connaitre leurs vrais enjeux locaux. Plusieurs citoyens de Montréal ont, par exemple, déploré l'absence de couverture journalistique de leur conseil d'arrondissement. Les personnes qui vivent en banlieue des grands centres urbains vivent le même phénomène (CPQ, 2008, p. 5).

Une étude de la firme Influence Communication démontre à ce titre qu'en 2016, c'est à Montréal que les médias offraient le moins de contenu local, soit $2 \%$, alors que la moyenne québécoise est à $11 \%$ (Influence Communication, 2016).

La sous-représentation régionale se traduit par ailleurs à travers un déficit d'attention sur le plan interrégional. Le Conseil de presse du Québec note : 
" [Les gens] déplorent aussi ne pas savoir ce qui se passe dans les autres régions du Québec » (CPQ, 2008, p. 6). Le rapport Payette a abondé dans le même sens : «Difficile pour un citoyen du Saguenay de savoir ce qui se passe en Gaspésie, et ce, même si les deux régions connaissent des problèmes similaires, notamment dans le secteur des ressources naturelles » (GTJAIQ, 2010a, p. 37).

La métropolisation de l'information réfère donc à la sous-représentation de la couverture médiatique, mais aussi à une couverture régionale négative. À cet égard, le Conseil de presse du Québec a noté une couverture déformée des régions, où une image folklorique de la réalité empreinte de préjugés sur les régions est beaucoup plus présente que les enjeux sociopolitiques régionaux: "Les médias ont toujours l'impression que les régions sont éloignées de Montréal, mais jamais le contraire » (CPQ, 2008, p. 17). Dans la même veine, le rapport Payette décrit:

La «montréalisation » de l'information a un impact sur la manière dont la région est perçue, et accentue le clivage entre les régions éloignées et les grands centres. «Fermeture d'usines, ouverture de la pêche au homard, concours de panaches d'orignaux, voilà en résumé le type de nouvelles régionales qui se rendent à l'antenne nationale. Les stéréotypes sont dommageables aussi pour nous, même si on ne les constate pas tous les jours », a résumé un participant à notre rencontre sur l'information en Gaspésie (GTJAIQ, 2010a, p. 34).

Le déséquilibre centre/périphérie concerne également l'accès à certaines ressources. L'accès à Internet en est un et, à cet égard, l'avantage des grands centres par rapport aux régions rurales est indéniable. «Frein au développement régional [...], l'absence d'Internet haute vitesse limite les transactions commerciales et agricoles, de même que l'accès aux informations gouvernementales. Les citoyens sont privés d'une source importante d'information régionale, nationale et internationale. Les enfants sont désavantagés dans leur formation. L'attrait de la région en est affecté »(CPQ, 2008, p. 21).

Le rapport Payette a également signalé la fracture numérique entre les régions éloignées et les grands centres en expliquant entre autres que les entreprises de télécommunication négligent d'investir dans les régions éloignées, car ce n'est pas rentable. Le rapport lie la fracture numérique à un déséquilibre entre inforiches et infopauvres (GTJAIQ, 2010a). Le Groupe de travail sur les collectivités rurales branchées avait recommandé au gouvernement du Québec d'assurer, à l'horizon 2015, « [qu'] Internet haute vitesse soit accessible sur la quasi-totalité du territoire rural habité de la province » (GTCRB, 2011, p. 27). Le taux de connexion à Internet haute vitesse augmente chaque année, mais le déséquilibre subsiste. L'enquête NETendances du CEFRIO indique que les régions souffrent plus des problèmes d'accessibilité et de connexion que les grands centres (CEFRIO, 2016). Les données du CRTC confirment les disparités concernant le choix des services Internet et certains prix d'autres services de communication entre les centres urbains et les collectivités rurales (CRTC, 2019).

Le déséquilibre en matière de répartition des ressources touche également le placement publicitaire gouvernemental. Lors de son témoignage à la Commission de la culture et de l'éducation, Sylvain Chamberland d'Arsenal Media a déploré que les ministères, les organismes et les sociétés d'État « surexposent la population métropolitaine et celle de la Capitale-Nationale aux messages gouvernementaux et négligent toujours les populations régionales » (Chamberland, 2019), malgré le fait que les statistiques démontrent que l'écoute des stations indépendantes dans les petits marchés du Québec représente près du tiers de l'écoute totale de la radio.

Avec la menace de la fermeture de plusieurs quotidiens régionaux du Groupe Capitales Médias, le spectre de la métropolisation de la presse écrite a ressurgi. La disparition potentielle d'un ou de plusieurs quotidiens signifierait de creuser davantage le vide informationnel régional, dans un contexte d'accessibilité médiatique déjà fragile en région. La Fédération des travailleurs et travailleuses du Québec a rappelé que la fermeture de journaux et de radios régionaux ainsi que les compressions dans les antennes télévisuelles locales contribuaient à la disparition des nouvelles locales, au profit de la centralisation du traitement de l'information dans les grands centres (FTQ, 2019). Dans son témoignage à la Commission de la culture et de l'éducation, la journaliste Marie-Ève Martel a évoqué les villes et les MRC, qui risquent de devenir des « déserts médiatiques ", à savoir "des lieux où se dérouleront tout plein de choses dont personne ne parlera » (Martel, 2019). 
Là où l'information n'existe pas ou n'existe plus, on évoque le désert. L'idée du désert renvoie à la sècheresse et à l'aridité d'un milieu, et se définit comme un territoire périphérique par rapport à un territoire plus nanti en ressources, même s'il peut comporter une ou plusieurs oasis locales. Lors des consultations qui ont mené au rapport Payette, un participant dénombrait environ 20 journalistes à Rimouski et Rivière-du-Loup dans le Bas-Saint-Laurent, « mais, dès qu'on sort de ces deux municipalités, on tombe dans un désert » (GTJAIQ, 2010b, p. 2).

Avec la crise médiatique majeure qui a occasionné des centaines de fermetures de médias locaux, la métaphore désertique a été mobilisée, car les territoires anciennement couverts par des médias sont délaissés, ce qui laisse un vide qui inquiète. $\mathrm{Si}$, au départ, le concept de « désert informationnel» a été minimalement défini comme «une communauté sans journal local» (Abernathy, 2018, p. 16), une définition plus étendue réfère à " une communauté, rurale ou urbaine, avec un accès limité à l'information crédible et complète qui alimente la démocratie à la base » (CISLM, 2019). Le désert informationnel désigne donc un territoire où l'information d'intérêt public est absente de l'écosystème social.

\section{Conclusion}

Tout le monde sait depuis un moment que l'économie des médias ne se porte pas bien du tout. L'industrie médiatique éprouve des défis majeurs et cherche des voies de sortie. La menace de fermeture de plusieurs quotidiens régionaux chez le Groupe Capitales Médias a créé un grand émoi avec la constatation des implications immenses de la disparition d'un tel groupe sur les salles de rédaction, sur les employés, sur les régions concernées et sur l'information locale. Le choc a provoqué un élan de solidarité en faveur des quotidiens et de leurs employés dans plusieurs régions. Un projet de coopérative soutenue par les employés a finalement été choisi par le tribunal pour relancer les six quotidiens.

L'objectif de cet article était de présenter les grands axes de réflexion sur l'évolution des médias régionaux et locaux, et nous avons identifié quatre types d'enjeux. Comme nous l'avons vu, la situation économique difficile des médias a attiré les projecteurs de l'actualité, et les enjeux économiques sont les plus urgents, eu égard aux difficultés inhérentes aux modèles d'affaires, particulièrement celui de la presse écrite. Dans le passé, malgré un large et constant débat au sein duquel des appels à l'action se sont fait entendre, les phénomènes de concentration et de convergence ont pris profondément racine dans l'écosystème médiatique québécois et canadien, phénomènes totalement ignorés par l'inaction des gouvernements fédéral et provincial.

Les pressions financières consécutives à la crise des médias ont forcé les gouvernements du Québec et du Canada à débloquer des fonds d'aide axés principalement sur la transition numérique et sur l'information régionale. "L'aide des gouvernements ne sera pas une panacée. Il n'y a pas de sortie de crise garantie. Néanmoins, cela devrait donner du temps et de l'espace aux médias traditionnels pour - et c'est ce qui est essentiel - s'adapter et évoluer vers des modèles d'affaires viables » (Brin et Descôteaux, 2018).

Les aides de l'État sont très sensibles et témoignent de l'urgence même de la situation. Cependant, l'État doit proposer une politique de soutien aux médias plus réfléchie en tenant compte des points de vue du milieu et des experts. Il ne s'agit pas uniquement de trouver un remède pour des maux urgents, mais c'est une occasion propice pour une véritable réflexion sur les politiques publiques de fonctionnement et de développement des médias, mais aussi de développement local et durable ainsi que de ruralité.

Les politiques publiques doivent tenir compte entre autres des déséquilibres entre les centres urbains et les collectivités rurales. Les propriétaires et les responsables des médias doivent se montrer aussi entreprenants, avec des mesures créatives, et miser sur l'innovation et la prise de risque pour réussir la transition numérique. Dans son ardent plaidoyer contre l'extinction des voix régionales, la journaliste Marie-Ève Martel soutient que «c'est en créant des contenus nouveaux, à valeur ajoutée, mais toujours centrés sur les préoccupations locales que les médias pourront tirer leur épingle du jeu » (2018, p. 158).

Comme principe, l'importance de l'existence des médias dans le fonctionnement d'une démocratie n'est plus à démontrer. Les municipalités doivent être redevables aux contribuables en acceptant le rôle de médiateur dévolu à la presse d'information. Les nouveaux outils technologiques ne doivent pas être un prétexte pour se soustraire à la transparence dans la divulgation de renseignements d'intérêt public. Les politiques publiques en matière d'accès à 
l'information et les politiques municipales sur la publicisation des débats publics doivent être cohérentes avec l'idéal démocratique et avec les principes de liberté de la presse et du droit du public à l'information. Les élus locaux ne peuvent pas affirmer que les médias sont nécessaires à la vie démocratique sans mettre en œuvre des politiques publiques conséquentes.

La situation des médias en région au Québec n’est pas très réjouissante. Les conséquences de la crise des médias et les déséquilibres inhérents à leur position périphérique par rapport aux médias des régions métropolitaines contribuent à leur fragilité et à la pauvreté de l'information locale. Sans médias locaux qui couvrent les affaires publiques et les projets de développement, les régions deviendront des déserts informationnels. L'espace de la ruralité, peu densifié et décentré, est celui qui apparait comme étant le plus susceptible de pâtir des situations de désert médiatique.

Face à la crise actuelle, les mesures d'aides publiques et les appels de solidarité envers la presse régionale traduisent une certaine prise de conscience des élus provinciaux et municipaux. Certains constats sont connus depuis longtemps, mais il faut des recherches scientifiques plus diversifiées sur différents enjeux médiatiques, par exemple le développement local. Des données précises peuvent informer les stratégies provinciales et municipales de développement local et durable. C'est une chose de taxer les géants du Web, mais c'en est une autre de penser à une taxation soucieuse d'atténuer au maximum les déséquilibres entre les grands centres et les régions rurales en matière de médiatisation locale et de ressources liées à la production de l'information locale.

\section{NOTES}

1 Le journal Les Affaires a été cédé en septembre 2019 au Groupe Contex, qui est dirigé par Pierre Marcoux, le fils du fondateur de TC Transcontinental.

2 Le coût de traitement par tonne est passé de 23,03 \$ en 2010 à 241,92 \$ en 2019 (Tétrault, 2019).

3 Durant la campagne électorale de 2019, le gouvernement fédéral a promis une taxe de 3\% pour les recettes des géants du web au Canada mais le Premier ministre Justin Trudeau a indiqué par la suite qu'il attendra les recommandations de l'Organisation de coopération et de développement économiques (OCDE).

4 L'échéance de cette aide du gouvernement du Québec est 2023-2024.

5 Le rapport Payette avait déjà recommandé « un crédit d'impôt remboursable à l'embauche de journalistes professionnels dans les entreprises en région» (GTJAIQ, 2010, p. 94). Devant la Commission de la culture et de l'éducation, TC Media et Hebdos Québec avaient recommandé un crédit d'impôt remboursable de $25 \%$ sur la masse salariale (TC Transcontinental, 2019; Hebdos Québec, 2019).

6 Cette loi stipule : «Une municipalité peut, par règlement, déterminer les modalités de publication de ses avis publics. Ces modalités peuvent différer selon le type d'avis, mais le règlement doit prévoir une publication sur Internet » (Assemblée nationale du Québec, 2017).

7 Municipalité régionale de comté (MRC).

8 Infoman est le titre d'une émission satirique présentée sur les ondes de Radio-Canada par l'animateur Jean-René Dufort. Des déclarations maladroites et intempestives et des faits cocasses enregistrés lors des conseils municipaux sont souvent repris dans cette émission. Martel (2018) donne l'exemple où le conseil municipal interdit l'enregistrement public après la diffusion des images à cette émission.

9 Acronyme qui renvoie aux personnes bispirituelles, lesbiennes, gaies, bisexuelles, transgenres, queers, en questionnement, intersexuées et asexuelles.

10 On peut penser à la plateforme La Fabrique culturelle de Télé-Québec.

11 Pour le rapport médias/territoires, voir Noyer, Raoul et Pailliart (2013); Noyer et Raoul (2011); et Proulx (1998).

12 Pour rappel, le groupe Gesca a vendu tous ses journaux régionaux au Groupe Capitales Médias en mars 2015.

\section{RÉFÉRENCES}

Abernathy, P. M. (2018). The Expanding News Desert. Chapel Hill, NC: Center for Innovative \& Sustainability in Local Media [University of North Carolina]. 
Alain, B. et Lalande, F. (2019, 23 août). Groupe Capitales Médias et propriété collective. Le Soleil. Repéré à https://www.lesoleil.com/opinions/point-de-vue/groupe-capitales-medias-et-propriete-collective-1a399748eb0ecdd3a7e09cc6f42792f2

Arsenal Media. (2019). Mémoire d'Arsenal Media à la Commission de la culture et de l'éducation de l'Assemblée nationale du Québec. Consultation sur l'avenir des médias d'information. Repéré à www.assnat.qc.ca

Assemblée nationale du Québec. (2017). Projet de loi nº122 (2017, chapitre 13). Loi visant principalement à reconnaître que les municipalités sont des gouvernements de proximité et à augmenter à ce titre leur autonomie et leurs pouvoirs, Québec, Éditeur officiel du Québec.

Assemblée nationale du Québec. (2019, 26 août). Journal des débats de la Commission de la culture et de l'éducation [témoignage], 45(23). Repéré à www.assnat.qc.ca

Association des radiodiffuseurs communautaires du Québec (ARCQ). (2016, mai). Mémoire déposé dans le cadre des consultations sur le renowvellement de la Politique culturelle du Québec. Repéré à https:/ /www.mcc.gouv.qc.ca/fileadmin/documents/

Politique-culturelle/Memoires__Metadonnees/Association_des_radiodiffuseurs_communautaires_du_Quebec.pdf

Baillargeon, S. (2016, 2 juin). Dépression régionale, Le Devoir, p. B10.

Bélanger, M.-F. (2017, 28 octobre). Webdiffuser les conseils municipaux, un défi pour de petites villes. Repéré à https:/ ici.radio-canada.ca/nouvelle/1063899/diffusion-reunion-conseil-ville-internet-transparence

Bibeau C. et Cyr C. (2019). Démocratie en péril : pour la prospérité de nos médias locaux et régionaux. Repéré à https://www.rocestrie.org/fr/ democratie-en-peril-pour-la-prosperite-de-nos-medias-locaux-et-regionaux?ssid=b371bwxcu72p664\&ssname=democratie_en_ peril.pdf

Bizimana, A.-J. et Kane, O. (2019). La presse au défi du numérique : une économie politique des médias régionaux au Québec. Les Cabiers du journalisme - Recherches, 2(3), 141-172. doi: 10.31188/CaJsm.2(3).2019.R141

Bourgault-Côté, G. (2017, 5 novrembre). Gare aux méchants journalistes. Le Devoir, p. B2.

Brin, C. et Descôteaux, B. (2018, 19 décembre). Aide aux médias canadiens : soutenir qui, pourquoi et comment? Options politiques. Repéré à https://policyoptions.irpp.org/fr/magazines/december-2018/aide-aux-medias-canadiens-soutenir-qui-pourquoi-etcomment/

Brin, C. et St-Pierre, M. (2013). Crise des médias et effectifs rédactionnels au Québec. Québec, QC : Centre d'études sur les médias.

Brunelle, A.-M. et Brin, C. (2019). L'information locale et régionale an Québec. Portrait du territoire 2011-2018 et perspectives citoyennes. Québec, QC : Centre d'études des médias.

Carignan, M.-È. (2012). L'état de l'information locale, régionale et nationale au Québec : Le point de vue des Premières Nations. Recherches amérindiennes au Québec, 42(1), 49-57. https://doi.org/10.7202/1023720ar

Centre facilitant la recherche et l'innovation dans les organisations (CEFRIO). (2016). Portrait numérique des foyers québécois. NETtendances 2016, 7(1). Repéré à cefrio.qc.ca

Chamberland, S. (2019, 28 août). Journal des débats de la Commission de la culture et de l'éducation [témoignage], 45(25). Repéré à http://www.assnat.qc.ca

Center for Innovative \& Sustainability in Local Media (CISLM). (2019). What exactly is a "News Desert"? Repéré à https://www.cislm.org/what-exactly-is-a-news-desert/

Coalition pour la pérennité de la presse d'information au Québec (CPPIQ). (2017). Les avis publics : un rempart pour notre démocratie. Mémoire déposé devant la Commission de l'aménagement du territoire dans le cadre des consultations sur le projet de loi 122, 22.02.17. Repéré à www.assnat.qc.ca

Comeau, P.-A. (2010). Loi sur l'accès à l'information et journalisme en milieu municipal. Avis rédigé à l'intention du Groupe de travail sur le journalisme et l'avenir de l'information. Repéré à https://www.mcc.gouv.qc.ca/fileadmin/documents/publications/media/PaulAndre_Comeau.pdf

Conférence régionale des élus de l'Abitibi-Témiscamingue (CREAT). (2011, 14 octobre). Mémoire dans le cadre de la consultation Pour une information au service de lintérêt public. Repéré à http://www.conferenceregionale.ca/documents/publications/memoire_ consultation_medias_20111018.pdf

Conseil de la culture de l'Estrie (CCE). (2006, octobre). Portrait-Secteur Communications. Repéré à http:/ www.cultureestrie.org/ fichiers/secteur-communications.pdf 
Conseil de la radiodiffusion et des télécommunications canadiennes (CRTC) (2012, 26 avril). Décision de radiodiffusion CRTC 2012-242. Repéré à crtc.gc.ca

Conseil de la radiodiffusion et des télécommunications canadiennes (CRTC). (2019). Rapport sur la surveillance des communications 2018. Repéré à crtc.gc.ca

Conseil de presse du Québec (CPQ). (2008). L'état de la situation médiatique du Québec : l'avis du public.

Repéré à https://conseildepresse.qc.ca/wp-content/uploads/2013/01/2008-11-10_etat-situation-mediatique_avis-du-public.pdf

Corriveau, R. et Sirois, G. (2012). L'information : la nécessaire perspective citoyenne. Québec, QC : Presses de l'Université du Québec.

Custeau, J. (2019, 20 septembre). Sherbrooke a versé plus de 78000 \$ aux géants du web en 2018. La Tribune.

Deloitte. (2014, 16 juin). L'impact économique de CBC/Radio-Canada en 2013. Un rapport produit pour CBC/Radio-Canada. Repéré à https:/ / site-cbc.radio-canada.ca/documents/vision/strategy/latest-studies/cbcradio-canada-impact-economiquedeloitte-2013.pdf

Demers, F. et Giroux, D. (2001, 14 juin). Information régionale et développement économique : Qu'en est-il dans votre région?. Québec, QC : Centre d'études sur les médias.

Enquête nationale sur les femmes et les filles autochtones disparues et assassinées (ENFFDA). (2019a). Réclamer notre pouvoir et notre place : le rapport final de l'Enquête nationale sur les femmes et les filles autochtones disparues et assassinées, 1a.

Repéré à https://www.mmiwg-ffada.ca/wp-content/uploads/2019/06/Rapport-final-volume-1a-1.pdf

Enquête nationale sur les femmes et les filles autochtones disparues et assassinées (ENFFDA). (2019b). Réclamer notre pouvoir et notre place : le rapport final de l'Enquête nationale sur les femmes et les filles autochtones disparues et assassinées, $1 \mathrm{~b}$.

Repéré à https://www.mmiwg-ffada.ca/wp-content/uploads/2019/06/Rapport-final-volume-1b.pdf

Fédération des travailleurs et travailleuses du Québec (FTQ). (2019, 16 mai). Mémoire de la Fédération des travailleurs et travailleuses du Québec (FTQ) sur l'avenir des médias d'information présenté à la Commission de la culture et de l'éducation.

Repéré à https://ftq.qc.ca/wp-content/uploads/2019/05/Memoire-FTQ-avenir-medias-Quebec_2019-05-16.pdf

Fédération professionnelle des journalistes du Québec (FPJQ). (2010, novembre). De nouvelles règles pour une meilleure circulation de l'information municipale an Québec Dossier noir. Mémoire présenté par la Fédération professionnelle des journalistes du Québec (FPJQ) au ministère des Affaires municipales, Régions et Occupation du territoire du Québec, à la Fédération québécoise des municipalités, à l'Union des municipalités du Québec et à la Commission d'accès à l'information.

Repéré à https://cdn.ca.yapla.com/company/CPY9xhmJrXC8hpGUQ5ssMX3n/asset/files/10-11-18_Dossier-noir.pdf

Fédération professionnelle des journalistes du Québec (FPJQ). (2013, mars). Vers un gouvernement ouvert. Mémoire de la Fédération professionnelle des journalistes du Québec sur la révision quinquennale de la Loi sur l'accès à l'information et la protection des renseignements personnels du Québec. Repéré à https://cdn.ca.yapla.com/ company/CPY9xhmJrXC8hpGUQ5ssMX3n/asset/files/12_Memoire_Acces_final.pdf

Fédération québécoise des municipalités (FQM). (2015). Résolution CA-01-09-2015/11. Position de la Fédération à l'égard de la question de l'enregistrement des séances de conseil municipal. Repéré à www.fqm.ca

Forum des politiques publiques. (2017, janvier). Le miroir éclaté. Nouvelles, démocratie et confiance dans l'ère numérique. Repéré à shatteredmirror.ca.

Forum des politiques publiques. (2018, septembre). Mind the Gaps. Quantifying the Decline of News Coverage in Canada. Repéré à shatteredmirror.ca

Gagnon, C. (2019a, 28 août). Journal des débats de la Commission de la culture et de l'éducation [témoignage], 45(25). Repéré à www.assnat.qc.ca

Gagnon, C. (2019b, 19 août). Nous allons continuer à vous informer et à vous servir! Le Soleil, p. 1-2.

Gilbert, A., Carrier, M. et Proulx M.-U. (2002, printemps). Les circuits d'information en région convergences et effet de milieu. Revue canadienne des sciences régionales, 25(1), 1-24. Repéré à http://www.cjrs-rcsr.org/archives/25-1/gilbert.pdf

Giroux, D. et Marcotte P. (2009). L'information régionale I- Le cas des quotidiens. Les cabiers-médias, 17, Centre d'études des médias.

Groupe de travail sur le journalisme et l'avenir de l'information au Québec (GTJAIQ).(2010a). L'information an Québec : un intérêt public, décembre. Repéré à www.mcc.gouv.qc.ca/fileadmin/documents/publications/media/rapport-Payette-2010.pdf 
Groupe de travail sur le journalisme et l'avenir de l'information au Québec (GTJAIQ). (2010b). Compte rendu de la rencontre sur l'information dans le Bas-Saint-Laurent tenue le 26 mai à Rimouski.

Groupe de travail sur les collectivités rurales branchées (GTCRB). (2011, 14 avril). Assurer le virage numérique des territoires ruraux : il y a ungence! Rapport final du Groupe de travail sur les collectivités rurales branchées. Repéré à https:/ / wnw. mamb.gouv.qc.cal fileadmin/publications/ developpement_territorial/ ruralite/groupes_travail/ rapport_collectivites_rurales_branchees.pdf

Hebdos Québec. (2019). Mémoire d'Hebdos Québec présenté à la Commission de la culture et de l'éducation. Repéré à www.assnat.qc.ca

Houde-Roy, L. (2017). Conseils municipaux. Passer l'action pour soutenir les journalistes. Le Trente, 25-27.

Influence Communication. (2016, 23 novembre), Analyse sur l'état de l'information locale au Québec présentée au Conseil provincial du secteur des communications (CPSC) du Syndicat canadien de la fonction publique (SCFP). Repéré à https:/ / scfp.qc.ca/wp-content/uploads/2017/04/nov_161123-Etude-Info-locale.pdf

Institut de la statistique du Québec. (2019). Statistiques principales des stations de télévision privées commerciales, régions administratives et ensemble du Québec. Repéré à http://www.stat.gouv.qc.ca

Islam, R. (2005). Gros plan : ce que disent les médias et pourquoi. Dans Banque mondiale, Le droit d’informer: Le rôle des médias dans le développement économique (p. 9-38). Paris : Éditions de Boeck Supérieur.

Jury, P. (2010, 16 février). La montréalisation des ondes. Le Droit, p. 12.

Lindgren, A., Corbett, J. et Jaingris H. (2017). Canada's local news "poverty". Policy Options, 23 janvier. Repéré à https://policyoptions.irpp.org/magazines/january-2017/canadas-local-news-poverty/

Lorrain, M. (2019, 28 août). Journal des débats de la Commission de la culture et de l'éducation [témoignage], 45 25). Repéré à www.assnat.qc.ca

Marcoux, P. (2019, 29 août). Journal des débats de la Commission de la culture et de l'éducation [témoignage], 45(26). Repéré à www.assnat.qc.ca

Martel, M.-È. (2018). Extinction de voix. Plaidoyer pour la sauvegarde de l'information régionale. Montréal, QC : Éditions Somme toute.

Martel, M.-È. (2019, 26 août). Journal des débats de la Commission de la culture et de l'éducation [témoignage], 45(23). Repéré à www.assnat.qc.ca

MCE Conseils. (2016). L'avenir de la presse et de linformation au Québec. Hypothèses de financement. Repéré à https://www.csn.qc.ca/ wp-content/uploads/2016/06/Rapport_financement-medias_juin-2016.pdf

Ministère de la Culture et des Communications (MCC).(2013). Évaluation : programme Aide au fonctionnement pour les médias communautaires. Repéré à https://www.mcc.gouv.qc.ca/fileadmin/documents/publications/EvaluationPAMEC_mars2013.pdf

Ministère des Affaires municipales, des Régions et de l'Occupation du territoire (MAMROT). (2013). Politique nationale de la ruralité 2014-2024. Québec : Gouvernement du Québec.

MPVradio. (2019). La publicité du gouvernement du Québec en région. Une question d'équité et de survie économique. Mémoire présenté dans le cadre de la Commission parlementaire sur l'avenir de l'information an Québec. Repéré à www.assnat.qc.ca

Normandin, P.-A. (2017, 30 août). Des citoyens veulent la fin de l'interdiction des caméras à LaSalle. La Presse. Repéré à https://www.lapresse.ca/actualites/montreal/201708/30/01-5128823-des-citoyens-veulent-la-fin-de-linterdiction-des-cameras-alasalle.php

Noyer, J. et Raoul, B. (2011). Le «travail territorial » des médias. Pour une approche conceptuelle et programmatique d'une notion. Études de communication, 37, 15-46. doi : 10.4000/edc.2933

Noyer, J., Raoul, B. et Pailliart I. (2013). Médias et territoires : l'espace public entre communication et imaginaire territorial. Villeneuve-d'Ascq, France : Presses Universitaires du Septentrion.

Olivier, F. (2019, 29 août). Journal des débats de la Commission de la culture et de l'éducation [témoignage], 45(26). Repéré à www.assnat.qc.ca

Owen, B. (2005). L’industrie des médias : les fondements économiques des communications de masse. Dans Banque mondiale (dir.), Le droit d'informer : Le rôle des médias dans le développement économique (p. 215-241). Paris : Éditions de Boeck Supérieur.

Proulx, M.-U. (1998). Médias dynamiques et territoires actifs. Problématique sur le rôle des médias locaux dans la vitalité économique de leur milieu. Sainte-Foy, QC : Centre d'études sur les médias.

80 
Sarrazin, P. (2019, 29 août). Les médias locaux: une nécessité communautaire et démocratique. La Voix de l'Est, p. 10.

TC Transcontinental. (2019, 27 février). Des solutions pour assurer l'avenir des médias fragilisés. Mémoire de TC Transcontinental dans le cadre du mandat d'initiative portant sur l'avenir des médias d'information de la Commission de la culture et de l'éducation (CCE).

Repéré à https://tctranscontinental.com/sites/default/files/2019-08/Me\%CC\%81moire\%20CCE_TC_2019-Final.pdf

Territoires innovants en économie sociale et solidaire (TIESS). (2014). Alliés pour le développement et le mieux-être des collectivités. Expérience innovante de partenariat entre l'économie sociale et le monde municipal. Station de radio CKBN. Repéré à http://www.tiess.ca/ documents/tiess_fiche8_RadioCKBN-web.pdf

Tétrault, D. (2019, 27 février). Recyclage du papier et médias : le point de rupture. Le Devoir, p. A7.

Trigone. (2014). Étude sur l'industrie du cinéma en région et sur les retombées économiques estimées d'un plan d'action concerté en cinéma court et créativité numérique au Saguenay-Lac-Saint-Jean. Rapport à la Ville de Saguenay.

Vailles, F. (2019, 29 août). Journaux régionaux: la retraite dans le tordeur. La Presse.ca. Repéré à https://www.lapresse.ca/affaires/ medias/201908/28/01-5239075-journaux-regionaux-la-retraite-dans-le-tordeur.php

Villeneuve, R. (2017, 13 avril). Lettre à Bernard Généreux, directeur général, Village de Val-David. Québec, QC : Bureau du commissaire aux plaintes. Repéré à https://www.mamh.gouv.qc.ca/fileadmin/publications/plainte_gestion_contractuelle/avis_ recommandations_directives/plaintes/2017/lettre_val_david_19_10_2017.pdf 